\title{
HYPERSURFACE FAMILIES WITH SMARANDACHE CURVES
} IN GALILEAN 4-SPACE

\author{
Mustafa ALTIN ${ }^{1}$, Ahmet KAZAN ${ }^{2}$, and H. Bayram KARADAĞ ${ }^{3}$ \\ ${ }^{1}$ Technical Sciences Vocational School, Bingöl University, Bingöl, TURKEY \\ ${ }^{2}$ Department of Computer Technologies, Doğanşehir Vahap Küçük Vocational School \\ of Higher Education, Malatya Turgut Özal University, Malatya, TURKEY \\ ${ }^{3}$ Department of Mathematics, Faculty of Arts and Sciences, İnönü University, \\ Malatya, TURKEY
}

\begin{abstract}
In this paper, we study the hypersurface families with Smarandache curves in 4-dimensional Galilean space $G_{4}$ and give the conditions for different Smarandache curves to be parameter and the curve which generates the Smarandache curves is geodesic on a hypersurface in $G_{4}$. Also, we investigate three types of marching-scale functions for one of these hypersurfaces and construct an example for it.
\end{abstract}

\section{PRELIMINARIES}

In physics, geodesics which are defined as a parallel transport of a tangent vector in a linear (affine) connection on the manifold $M$ are very important for general relativity. Because, the geodesic equation which is given with a set of initial conditions is very useful in theoretical foundations of general relativity. Also, in general, relativity gravity can be regarded as not a force but a consequence of a curved spacetime geometry where the source of curvature is the stress-energy tensor. For example, the path of a planet orbiting a star is the projection of a geodesic of the curved four-dimensional spacetime geometry around the star onto three-dimensional space.

Furthermore, as an alternative definition of a geodesic line can be defined as the shortest curve connecting two points on a manifold. A curve $\gamma(\nu)$ on a hypersurface $\varphi(\nu, \mu, \sigma)$ is geodesic iff the normal $N(\nu)$ of the curve $\gamma(\nu)$ and the normal $\eta\left(\nu, \mu_{0}, \sigma_{0}\right)$ of the hypersurface $\varphi(\nu, \mu, \sigma)$ at any point on the curve $\gamma(\nu)$ are parallel to each other and a curve $\gamma(\nu)$ on the hypersurface $\varphi(\nu, \mu, \sigma)$ is asymptotic iff

2020 Mathematics Subject Classification. 14J70, 53A35.

Keywords and phrases. Smarandache curve, hypersurface family, geodesic curve.

凶maltin@bingol.edu.tr-Corresponding author; ahmet.kazan@ozal.edu.tr; bayram.karadag@inonu.edu.tr

(D) 0000-0001-5544-5910; 0000-0002-1959-6102; 0000-0001-6474-877X. 
the normal $N(\nu)$ of the curve $\gamma(\nu)$ and the normal $\eta\left(\nu, \mu_{0}, \sigma_{0}\right)$ of the hypersurface $\varphi(\nu, \mu, \sigma)$ at any point on the curve $\gamma(\nu)$ are perpendicular.

The problem of constructing a family of surfaces from a given spatial geodesic curve firstly has been studied by Wang et al. in 2004 and in that study, the authors have derived a parametric representation for a surface pencil whose members share the same geodesic curve as an isoparametric curve [15. After this study, in 2008 the generalization of the Wangs' assumption to more general marching-scale functions has been given by Kasap et al 7]. By using these studies, the problem of finding a surface pencil from a given spatial asymptotic curve has been investigated in 4 and the necessary and sufficient condition for the given curve to be the asymptotic curve for the parametric surface has been stated in 11. Also, the problem of finding a hypersurface family from a given asymptotic curve in $R^{4}$ has been handled in 5 .

Surfaces with common geodesic and family of surface with a common null geodesic in Minkowski 3-space have been studied in [8] and [13, respectively.

The Galilean space $G_{3}$ is a Cayley-Klein space equipped with the metric of signature $(0,0,+,+)$. The absolute figure of the Galilean space consists of an ordered triple $\{\omega, f, I\}$ in which $\omega$ is the ideal (absolute) plane, $f$ is the line (absolute line) in $\omega$ and $I$ is the fixed elliptic involution of $f$.

In the Galilean $n$-space, there are just two types of vectors. A vector $u=$ $\left(u_{1}, u_{2}, \ldots, u_{n}\right)$ is said to be non-isotropic, if $u_{1} \neq 0$ and it is said to be isotropic otherwise.

If $u=\left(u_{1}, u_{2}, u_{3}, u_{4}\right), v=\left(v_{1}, v_{2}, v_{3}, v_{4}\right)$ and $w=\left(w_{1}, w_{2}, w_{3}, w_{4}\right)$ are three vectors in Galilean space $G_{4}$, then the Galilean scalar product of $x$ and $y$ is given by

$$
\langle u, v\rangle=\left\{\begin{array}{c}
u_{1} v_{1}, \text { if } u_{1} \neq 0 \text { or } v_{1} \neq 0 \\
u_{2} v_{2}+u_{3} v_{3}+u_{4} v_{4}, \text { if } u_{1}=0 \text { and } v_{1}=0
\end{array}\right.
$$

and for $e_{2}=(0,1,0,0), e_{3}=(0,0,1,0)$ and $e_{4}=(0,0,0,1)$, the Galilean cross product of $u, v$ and $w$ is defined by

$$
u \times v \times w=\left|\begin{array}{cccc}
0 & e_{2} & e_{3} & e_{4} \\
u_{1} & u_{2} & u_{3} & u_{4} \\
v_{1} & v_{2} & v_{3} & v_{4} \\
w_{1} & w_{2} & w_{3} & w_{4}
\end{array}\right| .
$$

Let $\gamma$ be an admissible curve of the class $C^{\infty}$ in $G_{4}$, parameterized by the invariant arc-length parameter $\nu$, given by

$$
\gamma(\nu)=(\nu, f(\nu), g(\nu), h(\nu))
$$

Then the Frenet frame is

$$
\begin{aligned}
& T(\nu)=\gamma^{\prime}(\nu)=\left(1, f^{\prime}(\nu), g^{\prime}(\nu), h^{\prime}(\nu)\right), \\
& N(\nu)=\frac{\gamma^{\prime \prime}(\nu)}{\kappa_{1}(\nu)}=\frac{1}{\kappa_{1}(\nu)}\left(0, f^{\prime \prime}(\nu), g^{\prime \prime}(\nu), h^{\prime \prime}(\nu)\right), \\
& B_{1}(\nu)=\frac{1}{\kappa_{2}(\nu)}\left(0,\left(\frac{f^{\prime \prime}(\nu)}{\kappa_{1}(\nu)}\right)^{\prime},\left(\frac{g^{\prime \prime}(\nu)}{\kappa_{1}(\nu)}\right)^{\prime},\left(\frac{h^{\prime \prime}(\nu)}{\kappa_{1}(\nu)}\right)^{\prime}\right), \\
& B_{2}(\nu)= \pm T(\nu) \times N(\nu) \times B_{1}(\nu)
\end{aligned}
$$


and the first, second and third curvatures of the curve $\gamma(\nu)$ are given by

$$
\begin{aligned}
& \kappa_{1}(\nu)=\sqrt{f^{\prime \prime}(\nu)^{2}+g^{\prime \prime}(\nu)^{2}+h^{\prime \prime}(\nu)^{2}}, \\
& \kappa_{2}(\nu)=\sqrt{\left\langle N^{\prime}(\nu), N^{\prime}(\nu)\right\rangle}, \\
& \kappa_{3}(\nu)=\left\langle B_{1}^{\prime}(\nu), B_{2}(\nu)\right\rangle,
\end{aligned}
$$

respectively and where $T, N, B_{1}$ and $B_{2}$ are called tangent vector, principal normal vector, first binormal vector and second binormal vector of $\gamma(\nu)$. We must note that, throughout this study, we will assume that $\kappa_{1}(\nu) \neq 0$ and $\kappa_{2}(\nu) \neq 0$ at everywhere.

Also, Frenet formulas are given by

$$
\begin{aligned}
& T^{\prime}(\nu)=\kappa_{1}(\nu) N(\nu), \\
& N^{\prime}(\nu)=\kappa_{2}(\nu) B_{1}(\nu), \\
& B_{1}^{\prime}(\nu)=-\kappa_{2}(\nu) N(\nu)+\kappa_{3}(\nu) B_{2}(\nu), \\
& B_{2}^{\prime}(\nu)=-\kappa_{3}(\nu) B_{1}(\nu) .
\end{aligned}
$$

The equation of a hypersurface in $G_{4}$ can be given by the parametrization

$$
\varphi(\nu, \mu, \sigma)=\left((\varphi(\nu, \mu, \sigma))_{1},(\varphi(\nu, \mu, \sigma))_{2},(\varphi(\nu, \mu, \sigma))_{3},(\varphi(\nu, \mu, \sigma))_{4}\right),
$$

where $(\varphi(\nu, \mu, \sigma))_{i} \in C^{3}, i=1,2,3,4$. The normal of this hypersurface is calculated as follows

$$
\eta(\nu, \mu, \sigma)=\varphi_{\nu} \times \varphi_{\mu} \times \varphi_{\sigma},
$$

where $\varphi_{i}=\frac{\partial \varphi(\nu, \mu, \sigma)}{\partial i}, i \in\{\nu, \mu, \sigma\}$.

For more information about 4-dimensional Galilean space, we refer to 6], 11], [12, 16], 17] and etc.

If $\gamma(\nu)$ is a isoparametric curve on the hypersurface $\varphi(\nu, \mu, \sigma)$, then there exists a pair of parameters $\mu_{0} \in\left[T_{1}, T_{2}\right]$ and $\sigma_{0} \in\left[M_{1}, M_{2}\right]$, such that $\gamma(\nu)=\varphi\left(\nu, \mu_{0}, \sigma_{0}\right)$.

If the curve is both an asymptotic and parameter (isoparametric) curve on $\varphi$, then it is called isoasymptotic on the hypersurface $\varphi$. Similarly, if the curve is both a geodesic and parameter (isoparametric) curve on the hypersurface $\varphi$, then it is called isogeodesic on the hypersurface $\varphi$.

On the constructions of surface families with common geodesic and asymptotic curves in Galilean Space $G_{3}$ and an approach for hypersurface family with common geodesic curve in the Galilean Space $G_{4}$ have been handled in [9], [10] and 11, respectively.

On the other hand, the geometry of Smarandache curves has been very popular topic for differential geometers, recently. Let $\gamma(\nu)$ be an admissible curve in $G_{4}$ and $\left\{T, N, B_{1}, B_{2}\right\}$ be its moving Frenet frame. Then $T N, T B_{1}, T B_{2}, N B_{1}, N B_{2}, B_{1} B_{2}$, $T N B_{1}, T N B_{2}, T B_{1} B_{2}, N B_{1} B_{2}$ and $T N B_{1} B_{2}$-Smarandache curves are defined by $r_{T N}=\frac{T+N}{\|T+N\|}, r_{T B_{1}}=\frac{T+B_{1}}{\left\|T+B_{1}\right\|}, r_{T B_{2}}=\frac{T+B_{2}}{\left\|T+B_{2}\right\|}, r_{N B_{1}}=\frac{N+B_{1}}{\left\|N+B_{1}\right\|}, r_{N B_{2}}=\frac{N+B_{2}}{\left\|N+B_{2}\right\|}$, $r_{B_{1} B_{2}}=\frac{B_{1}+B_{2}}{\left\|B_{1}+B_{2}\right\|}, r_{T N B_{1}}=\frac{T+N+B_{1}}{\left\|T+N+B_{1}\right\|}, r_{T N B_{2}}=\frac{T+N+B_{2}}{\left\|T+N+B_{2}\right\|}, r_{T B_{1} B_{2}}=\frac{T+B_{1}+B_{2}}{\left\|T+B_{1}+B_{2}\right\|}$, $r_{N B_{1} B_{2}}=\frac{N+B_{1}+B_{2}}{\left\|N+B_{1}+B_{2}\right\|}, r_{T N B_{1} B_{2}}=\frac{T+N+B_{1}+B_{2}}{\left\|T+N+B_{1}+B_{2}\right\|}$, respectively.

The problem of constructing a family of surfaces from a given some special Smarandache asymptotic curves in Euclidean 3-space has been analyzed in [14] and 
surfaces using Smarandache asymptotic curves in Galilean space have been studied in $[2]$.

In the present study, we investigate the hypersurface families with Smarandache curves in 4-dimensional Galilean space $G_{4}$.

\section{HYPERSURFACE FAMILIES WITH SMARANDACHE CURVES IN 4-DIMENSIONAL GALILEAN SPACE $G_{4}$}

Let $\varphi(\nu, \mu, \sigma)$ be a parametric hypersurface which is defined by a given curve $\gamma(\nu)$ as follows

$$
\varphi(\nu, \mu, \sigma)=\gamma(\nu)+\left[\begin{array}{c}
x(\nu, \mu, \sigma) T(\nu)+y(\nu, \mu, \sigma) N(\nu) \\
+z(\nu, \mu, \sigma) B_{1}(\nu)+m(\nu, \mu, \sigma) B_{2}(\nu)
\end{array}\right],
$$

where $L_{1} \leq \nu \leq L_{2}, T_{1} \leq \mu \leq T_{2}$ and $M_{1} \leq \sigma \leq M_{2}$. Also, $x(\nu, \mu, \sigma), y(\nu, \mu, \sigma)$, $z(\nu, \mu, \sigma)$ and $m(\nu, \mu, \sigma)$ which are the values of the marching-scale functions indicate and the values of these functions are $C^{1}$-functions and $\left\{T, N, B_{1}, B_{2}\right\}$ is the Frenet frame associated with the curve $\gamma$ in $G_{4}$.

Throughout this study, for simplicity, we will denote $x(\nu, \mu, \sigma)=x, x\left(\nu, \mu_{0}, \sigma_{0}\right)$ $=x_{0}, \frac{\partial x(\nu, \mu, \sigma)}{\partial \nu}=x_{\nu}, \frac{\partial x(\nu, \mu, \sigma)}{\partial \mu}=x_{\mu}, \frac{\partial x(\nu, \mu, \sigma)}{\partial \sigma}=x_{\sigma}$ and $\left.\frac{\partial x(\nu, \mu, \sigma)}{\partial \nu}\right|_{\left(\nu, \mu_{0}, \sigma_{0}\right)}=\left(x_{v}\right)_{0}$, $\left.\frac{\partial x(\nu, \mu, \sigma)}{\partial \mu}\right|_{\left(\nu, \mu_{0}, \sigma_{0}\right)}=\left(x_{\mu}\right)_{0},\left.\frac{\partial x(\nu, \mu, \sigma)}{\partial v}\right|_{\left(\nu, \mu_{0}, \sigma_{0}\right)}=\left(x_{\sigma}\right)_{0}$. Similar abbreviations for $y(\nu, \mu, \sigma), z(\nu, \mu, \sigma)$ and $m(\nu, \mu, \sigma)$ will be used, too.

CASE 1.

In this case, by taking the $T N$-Smarandache curve of $\gamma(\nu)$ instead of the curve $\gamma(\nu)$ in $(9)$, let us define a parametric hypersurface $\varphi_{T N}(\nu, \mu, \sigma)$ which is given with the aid of the $T N$-Smarandache curve of $\gamma(\nu)$ and the Frenet vectors of the curve $\gamma(\nu)$ as follows

$$
\varphi_{T N}(\nu, \mu, \sigma)=r_{T N}(\nu)+\left[\begin{array}{c}
x(\nu, \mu, \sigma) T(\nu)+y(\nu, \mu, \sigma) N(\nu) \\
+z(\nu, \mu, \sigma) B_{1}(\nu)+m(\nu, \mu, \sigma) B_{2}(\nu)
\end{array}\right] .
$$

From 10 , we have

$$
\begin{aligned}
\left(\varphi_{T N}\right)_{\nu} & =x_{\nu} T+\left(\kappa_{1}+x \kappa_{1}+y_{\nu}-z \kappa_{2}\right) N \\
& +\left(\kappa_{2}+y \kappa_{2}+z_{\nu}-m \kappa_{3}\right) B_{1}+\left(m_{\nu}+z \kappa_{3}\right) B_{2}, \\
\left(\varphi_{T N}\right)_{\mu} & =x_{\mu} T+y_{\mu} N+z_{\mu} B_{1}+m_{\mu} B_{2}, \\
\left(\varphi_{T N}\right)_{\sigma} & =x_{\sigma} T+y_{\sigma} N+z_{\sigma} B_{1}+m_{\sigma} B_{2},
\end{aligned}
$$

where we denote $\frac{\partial \varphi_{T N}(\nu, \mu, \sigma)}{\partial \nu}=\left(\varphi_{T N}\right)_{\nu}, \frac{\partial \varphi_{T N}(\nu, \mu, \sigma)}{\partial \mu}=\left(\varphi_{T N}\right)_{\mu}, \frac{\partial \varphi_{T N}(\nu, \mu, \sigma)}{\partial \sigma}=$ $\left(\varphi_{T N}\right)_{\sigma}$.

Thus, if we use (11) in (8), by obtaining the normal of the hypersurface 10$]$, we can state the following theorem:

Theorem 1. $\gamma(\nu)$ is not a geodesic curve where TN-Smarandache curve $r_{T N}$ of the curve $\gamma(\nu)$ is isoparametric on the hypersurface $\varphi_{N B_{1}}(\nu, \mu, \sigma)$ in $G_{4}$. 
Proof. If $T N$-Smarandache curve is a isoparametric curve on $\varphi_{T N}(\nu, \mu, \sigma)$, then there exists a pair of parameters $\mu=\mu_{0}$ and $\sigma=\sigma_{0}$ such that

$$
\varphi_{T N}\left(\nu, \mu_{0}, \sigma_{0}\right)=T(\nu)+N(\nu),
$$

that is

$$
x\left(\nu, \mu_{0}, \sigma_{0}\right)=y\left(\nu, \mu_{0}, \sigma_{0}\right)=z\left(\nu, \mu_{0}, \sigma_{0}\right)=m\left(\nu, \mu_{0}, \sigma_{0}\right)=0,
$$

where $L_{1} \leq \nu \leq L_{2}, T_{1} \leq \mu_{0} \leq T_{2}$ and $M_{1} \leq \sigma_{0} \leq M_{2}$. Here we must note that, from 12 , we have

$$
x_{v}\left(\nu, \mu_{0}, \sigma_{0}\right)=y_{v}\left(\nu, \mu_{0}, \sigma_{0}\right)=z_{v}\left(\nu, \mu_{0}, \sigma_{0}\right)=m_{v}\left(\nu, \mu_{0}, \sigma_{0}\right)=0 .
$$

So, from (2), 11), 12 and (13), the normal of the hypersurface 10 for $\mu=\mu_{0}$ and $\sigma=\sigma_{0}$ is obtained as

$$
\begin{aligned}
\eta_{T N}\left(\nu, \mu_{0}, \sigma_{0}\right) & =\left(\eta_{T N}\right)_{1}\left(\nu, \mu_{0}, \sigma_{0}\right) T(\nu)+\left(\eta_{T N}\right)_{2}\left(\nu, \mu_{0}, \sigma_{0}\right) N(\nu) \\
& +\left(\eta_{T N}\right)_{3}\left(\nu, \mu_{0}, \sigma_{0}\right) B_{1}(\nu)+\left(\eta_{T N}\right)_{4}\left(\nu, \mu_{0}, \sigma_{0}\right) B_{2}(\nu),
\end{aligned}
$$

where

$$
\begin{aligned}
\left(\eta_{T N}\right)_{1}\left(\nu, \mu_{0}, \sigma_{0}\right) & =0, \\
\left(\eta_{T N}\right)_{2}\left(\nu, \mu_{0}, \sigma_{0}\right) & =\kappa_{2}\left(\left(m_{\sigma}\right)_{0}\left(x_{\mu}\right)_{0}-\left(x_{\sigma}\right)_{0}\left(m_{\mu}\right)_{0}\right), \\
\left(\eta_{T N}\right)_{3}\left(\nu, \mu_{0}, \sigma_{0}\right) & =\kappa_{1}\left(\left(m_{\mu}\right)_{0}\left(x_{\sigma}\right)_{0}-\left(x_{\mu}\right)_{0}\left(m_{\sigma}\right)_{0}\right), \\
\left(\eta_{T N}\right)_{4}\left(\nu, \mu_{0}, \sigma_{0}\right) & =\kappa_{1}\left(\left(x_{\mu}\right)_{0}\left(z_{\sigma}\right)_{0}-\left(z_{\mu}\right)_{0}\left(x_{\sigma}\right)_{0}\right) \\
& +\kappa_{2}\left(\left(y_{\mu}\right)_{0}\left(x_{\sigma}\right)_{0}-\left(x_{\mu}\right)_{0}\left(y_{\sigma}\right)_{0}\right) .
\end{aligned}
$$

Also, from the definition of a given curve $\gamma(\nu)$ on the hypersurface $\varphi(\nu, \mu, \sigma)$ to be geodesic, it must be

$$
\left(\eta_{T N}\right)_{2}\left(\nu, \mu_{0}, \sigma_{0}\right) \neq 0
$$

and

$$
\left(\eta_{T N}\right)_{3}\left(\nu, \mu_{0}, \sigma_{0}\right)=\left(\eta_{T N}\right)_{4}\left(\nu, \mu_{0}, \sigma_{0}\right)=0 .
$$

So, from 15) the curve $\gamma(\nu)$ is a geodesic on the hypersurface $\varphi_{T N}(\nu, \mu, \sigma)$ in $G_{4}$ if

$$
\left\{\begin{array}{l}
x\left(\nu, \mu_{0}, \sigma_{0}\right)=y\left(\nu, \mu_{0}, \sigma_{0}\right)=z\left(\nu, \mu_{0}, \sigma_{0}\right)=m\left(\nu, \mu_{0}, \sigma_{0}\right)=0 \\
\kappa_{1}=0, \kappa_{2} \neq 0 \\
\left(y_{\mu}\right)_{0}\left(x_{\sigma}\right)_{0}=\left(x_{\mu}\right)_{0}\left(y_{\sigma}\right)_{0},\left(m_{\mu}\right)_{0}\left(x_{\sigma}\right)_{0} \neq\left(x_{\mu}\right)_{0}\left(m_{\sigma}\right)_{0}
\end{array}\right.
$$

satisfied, where $\nu \in\left[L_{1}, L_{2}\right], \mu_{0} \in\left[T_{1}, T_{2}\right], \sigma_{0} \in\left[M_{1}, M_{2}\right]$. Since $\kappa_{1}(v) \neq 0$, the proof completes.

\section{CASE 2.}

Here, by taking the $N B_{2}$-Smarandache curve of $\gamma(\nu)$ instead of the curve $\gamma(\nu)$ in 9 , let us define a parametric hypersurface $\varphi_{N B_{2}}(\nu, \mu, \sigma)$ which is given with the aid of the $\mathrm{NB}_{2}$-Smarandache curve of $\gamma(\nu)$ and the Frenet vectors of the curve $\gamma(\nu)$ as follows

$$
\varphi_{N B_{2}}(\nu, \mu, \sigma)=r_{N B_{2}}(\nu)+\left[\begin{array}{c}
x(\nu, \mu, \sigma) T(\nu)+y(\nu, \mu, \sigma) N(\nu) \\
+z(\nu, \mu, \sigma) B_{1}(\nu)+m(\nu, \mu, \sigma) B_{2}(\nu)
\end{array}\right] .
$$


If $N B_{2}$-Smarandache curve of the curve $\gamma(\nu)$ is a isoparametric curve on a hypersurface $\varphi_{N B_{2}}(\nu, \mu, \sigma)$ in $G_{4}$ for $\mu=\mu_{0}$ and $\sigma=\sigma_{0}$, then from (8), the normal of this hypersurface is

$$
\begin{aligned}
\eta_{N B_{2}}\left(\nu, \mu_{0}, \sigma_{0}\right) & =\left(\eta_{N B_{2}}\right)_{1}\left(\nu, \mu_{0}, \sigma_{0}\right) T(\nu)+\left(\eta_{N B_{2}}\right)_{2}\left(\nu, \mu_{0}, \sigma_{0}\right) N(\nu) \\
& +\left(\eta_{N B_{2}}\right)_{3}\left(\nu, \mu_{0}, \sigma_{0}\right) B_{1}(\nu)+\left(\eta_{N B_{2}}\right)_{4}\left(\nu, \mu_{0}, \sigma_{0}\right) B_{2}(\nu),
\end{aligned}
$$

where

$$
\begin{aligned}
& \left(\eta_{N B_{2}}\right)_{1}\left(\nu, \mu_{0}, \sigma_{0}\right)=0 \\
& \left(\eta_{N B_{2}}\right)_{2}\left(\nu, \mu_{0}, \sigma_{0}\right)=\left(\frac{\kappa_{2}-\kappa_{3}}{\sqrt{2}}\right)\left(\left(x_{\mu}\right)_{0}\left(m_{\sigma}\right)_{0}-\left(m_{\mu}\right)_{0}\left(x_{\sigma}\right)_{0}\right), \\
& \left(\eta_{N B_{2}}\right)_{3}\left(\nu, \mu_{0}, \sigma_{0}\right)=0, \\
& \left(\eta_{N B_{2}}\right)_{4}\left(\nu, \mu_{0}, \sigma_{0}\right)=\left(\frac{\kappa_{3}-\kappa_{2}}{\sqrt{2}}\right)\left(\left(y_{\sigma}\right)_{0}\left(x_{\mu}\right)_{0}-\left(x_{\sigma}\right)_{0}\left(y_{\mu}\right)_{0}\right) .
\end{aligned}
$$

Theorem 2. $\gamma(\nu)$ is a geodesic curve where $N B_{2}$-Smarandache curve $r_{N B_{2}}$ of the curve $\gamma(\nu)$ is isoparametric on the hypersurface $\varphi_{N B_{2}}(\nu, \mu, \sigma)$ in $G_{4}$ if the conditions

$$
\left\{\begin{array}{l}
x\left(\nu, \mu_{0}, \sigma_{0}\right)=y\left(\nu, \mu_{0}, \sigma_{0}\right)=z\left(\nu, \mu_{0}, \sigma_{0}\right)=m\left(\nu, \mu_{0}, \sigma_{0}\right)=0, \\
\kappa_{2} \neq \kappa_{3}, \\
\left(y_{\mu}\right)_{0}\left(x_{\sigma}\right)_{0}=\left(x_{\mu}\right)_{0}\left(y_{\sigma}\right)_{0},\left(m_{\mu}\right)_{0}\left(x_{\sigma}\right)_{0} \neq\left(x_{\mu}\right)_{0}\left(m_{\sigma}\right)_{0}
\end{array}\right.
$$

are satisfied. Here, $\nu \in\left[L_{1}, L_{2}\right], \mu_{0} \in\left[T_{1}, T_{2}\right], \sigma_{0} \in\left[M_{1}, M_{2}\right]$.

Proof. If $x\left(\nu, \mu_{0}, \sigma_{0}\right)=y\left(\nu, \mu_{0}, \sigma_{0}\right)=z\left(\nu, \mu_{0}, \sigma_{0}\right)=m\left(\nu, \mu_{0}, \sigma_{0}\right)=0$ satisfies for a pair of parameters $\mu=\mu_{0}$ and $\sigma=\sigma_{0}$ on hypersurface $\varphi_{N B_{2}}(\nu, \mu, \sigma)$, then $N B_{2}$-Smarandache curve is a isoparametric curve such that

$$
\varphi_{N B_{2}}\left(\nu, \mu_{0}, \sigma_{0}\right)=\frac{N(\nu)+B_{2}(\nu)}{\sqrt{2}},
$$

where $L_{1} \leq \nu \leq L_{2}, T_{1} \leq \mu_{0} \leq T_{2}$ and $M_{1} \leq \sigma_{0} \leq M_{2}$.

Also, from the definition of a given curve $\gamma(\nu)$ on the hypersurface $\varphi(\nu, \mu, \sigma)$ to be geodesic where $\mathrm{NB}_{2}$-Smarandache curve $r_{\mathrm{NB}_{2}}$ of the curve $\gamma(\nu)$ is isoparametric, it must be

and

$$
\left(\eta_{N B_{1}}\right)_{2}\left(\nu, \mu_{0}, \sigma_{0}\right) \neq 0
$$

$$
\left(\eta_{N B_{1}}\right)_{3}\left(\nu, \mu_{0}, \sigma_{0}\right)=\left(\eta_{N B_{1}}\right)_{4}\left(\nu, \mu_{0}, \sigma_{0}\right)=0
$$

So, using these conditions with $(19)$ in $(18)$, the proof completes.

For the purposes of simplification and better analysis, Wang et al. have studied the case when the marching-scale functions can be decomposed into two factors in Euclidean 3-space. The factor-decomposition form possesses an evident advantage: the designer can select different sets of functions to adjust the shape of the surface until they are gratified with the design, and the resulting surface is guaranteed to belong to the isogeodesic surface pencil with the curve as the common geodesic 15]. Also in [3] and [11, the three types of the marching-scale function which have three parameters have been studied in 4-dimensional Galilean and Euclidean spaces, 
respectively. In this study, we have used the marching-scale functions which have been given in these studies. Now, for simplicity, let we investigate three types of marching-scale functions for this hypersurface.

\section{Marching-scale functions of type 1}

Let us choose

$$
\left\{\begin{array}{l}
x(\nu, \mu, \sigma)=p(\nu) X(\mu, \sigma) \\
y(\nu, \mu, \sigma)=q(\nu) Y(\mu, \sigma) \\
z(\nu, \mu, \sigma)=w(\nu) Z(\mu, \sigma) \\
m(\nu, \mu, \sigma)=l(\nu) M(\mu, \sigma)
\end{array}\right.
$$

where $L_{1} \leq \nu \leq L_{2}, T_{1} \leq \mu \leq T_{2}, M_{1} \leq \sigma \leq M_{2} ; p(\nu), q(\nu), w(\nu), l(\nu), X(\mu, \sigma)$, $Y(\mu, \sigma), Z(\mu, \sigma), M(\mu, \sigma) \in C^{1}$ and $p(\nu), q(\nu), w(\nu), l(\nu), \forall \nu \in\left[L_{1}, L_{2}\right]$ are not identically zero. By using 19 , if the conditions

$$
\left\{\begin{array}{l}
X\left(\mu_{0}, \sigma_{0}\right)=Y\left(\mu_{0}, \sigma_{0}\right)=Z\left(\mu_{0}, \sigma_{0}\right)=M\left(\mu_{0}, \sigma_{0}\right)=0 \\
\kappa_{2} \neq \kappa_{3}, \\
\frac{\partial Y\left(\mu_{0}, \sigma_{0}\right)}{\partial \mu} \frac{\partial X\left(\mu_{0}, \sigma_{0}\right)}{\partial \sigma}=\frac{\partial X\left(\mu_{0}, \sigma_{0}\right)}{\partial \mu} \frac{\partial Y\left(\mu_{0}, \sigma_{0}\right)}{\partial \sigma} \\
\frac{\partial M\left(\mu_{0}, \sigma_{0}\right)}{\partial \mu} \frac{\partial X\left(\mu_{0}, \sigma_{0}\right)}{\partial \sigma} \neq \frac{\partial X\left(\mu_{0}, \sigma_{0}\right)}{\partial \mu} \frac{\partial M\left(\mu_{0}, \sigma_{0}\right)}{\partial \sigma}
\end{array}\right.
$$

satisfy, then $\gamma(\nu)$ is a geodesic curve where $N B_{2}$-Smarandache curve $r_{N B_{2}}$ of the curve $\gamma(\nu)$ is isoparametric on the hypersurface $\varphi_{N B_{2}}(\nu, \mu, \sigma)$ in $G_{4}$. Here, $\mu_{0} \in$ $\left[T_{1}, T_{2}\right], \sigma_{0} \in\left[M_{1}, M_{2}\right]$.

\section{Marching-scale functions of type 2}

If we take

$$
\left\{\begin{array}{c}
x(\nu, \mu, \sigma)=p(\nu, \mu) X(\sigma) \\
y(\nu, \mu, \sigma)=q(\nu, \mu) Y(\sigma) \\
z(\nu, \mu, \sigma)=w(\nu, \mu) Z(\sigma) \\
m(\nu, \mu, \sigma)=l(\nu, \mu) M(\sigma)
\end{array}\right.
$$

where $L_{1} \leq \nu \leq L_{2}, T_{1} \leq \mu \leq T_{2}, M_{1} \leq \sigma \leq M_{2}$ and $p(\nu, \mu), q(\nu, \mu), w(\nu, \mu)$, $l(\nu, \mu), X(\mu), Y(\mu), Z(\mu), M(\mu) \in C^{1}$, by using 19$)$, if the conditions

$$
\left\{\begin{array}{l}
p\left(\nu, \mu_{0}\right) X\left(\sigma_{0}\right)=q\left(\nu, \mu_{0}\right) Y\left(\sigma_{0}\right)=w\left(\nu, \mu_{0}\right) Z\left(\sigma_{0}\right)=l\left(\nu, \mu_{0}\right) M\left(\sigma_{0}\right)=0 \\
\kappa_{2} \neq \kappa_{3} \\
\frac{\partial q\left(\nu, \mu_{0}\right)}{\partial \mu} Y\left(\sigma_{0}\right) p\left(\nu, \mu_{0}\right) \frac{\partial X\left(\sigma_{0}\right)}{\partial \sigma}=\frac{\partial p\left(\nu, \mu_{0}\right)}{\partial \mu} X\left(\sigma_{0}\right) q\left(\nu, \mu_{0}\right) \frac{\partial Y\left(\sigma_{0}\right)}{\partial \sigma} \\
\frac{\partial l\left(\nu, \mu_{0}\right)}{\partial \mu} M\left(\sigma_{0}\right) p\left(\nu, \mu_{0}\right) \frac{\partial X\left(\sigma_{0}\right)}{\partial \sigma} \neq \frac{\partial p\left(\nu, \mu_{0}\right)}{\partial \mu} X\left(\sigma_{0}\right) l\left(\nu, \mu_{0}\right) \frac{\partial M\left(\sigma_{0}\right)}{\partial \sigma}
\end{array}\right.
$$

satisfy, then $\gamma(\nu)$ is a geodesic curve where $N B_{2}$-Smarandache curve $r_{N B_{2}}$ of the curve $\gamma(\nu)$ is isoparametric on the hypersurface $\varphi_{N B_{2}}(\nu, \mu, \sigma)$ in $G_{4}$. Here, $\mu_{0} \in$ $\left[T_{1}, T_{2}\right], \sigma_{0} \in\left[M_{1}, M_{2}\right]$.

Marching-scale functions of type 3 
For

$$
\left\{\begin{array}{l}
x(\nu, \mu, \sigma)=p(\nu, \sigma) X(\mu), \\
y(\nu, \mu, \sigma)=q(\nu, \sigma) Y(\mu), \\
z(\nu, \mu, \sigma)=w(\nu, \sigma) Z(\mu), \\
m(\nu, \mu, \sigma)=l(\nu, \sigma) M(\mu),
\end{array}\right.
$$

where $L_{1} \leq \nu \leq L_{2}, T_{1} \leq \mu \leq T_{2}, M_{1} \leq \sigma \leq M_{2}$ and $p(\nu, \sigma), q(\nu, \sigma), w(\nu, \sigma)$, $l(\nu, \sigma), X(\mu), Y(\mu), Z(\mu), M(\mu) \in C^{1}$, from (19), if the conditions

$$
\left\{\begin{array}{l}
p\left(\nu, \sigma_{0}\right) X\left(\mu_{0}\right)=q\left(\nu, \sigma_{0}\right) Y\left(\mu_{0}\right)=w\left(\nu, \sigma_{0}\right) Z\left(\mu_{0}\right)=l\left(\nu, \sigma_{0}\right) M\left(\mu_{0}\right)=0 \\
\kappa_{2} \neq \kappa_{3} \\
\frac{\partial Y\left(\mu_{0}\right)}{\partial \mu} q\left(\nu, \sigma_{0}\right) \frac{\partial p\left(\nu, \sigma_{0}\right)}{\partial \sigma} X\left(\mu_{0}\right)=\frac{\partial X\left(\mu_{0}\right)}{\partial \mu} p\left(\nu, \sigma_{0}\right) \frac{\partial q\left(\nu, \sigma_{0}\right)}{\partial \sigma} Y\left(\mu_{0}\right) \\
\frac{\partial M\left(\mu_{0}\right)}{\partial \mu} l\left(\nu, \sigma_{0}\right) \frac{\partial p\left(\nu, \sigma_{0}\right)}{\partial \sigma} X\left(\mu_{0}\right) \neq \frac{\partial X\left(\mu_{0}\right)}{\partial \mu} p\left(\nu, \sigma_{0}\right) \frac{\partial l\left(\nu, \sigma_{0}\right)}{\partial \sigma} M\left(\mu_{0}\right)
\end{array}\right.
$$

satisfy, then $\gamma(\nu)$ is a geodesic curve where $N B_{2}$-Smarandache curve $r_{N B_{2}}$ of the curve $\gamma(\nu)$ is isoparametric on the hypersurface $\varphi_{N B_{2}}(\nu, \mu, \sigma)$ in $G_{4}$. Here, $\mu_{0} \in\left[T_{1}, T_{2}\right], \sigma_{0} \in\left[M_{1}, M_{2}\right]$.

Now, let we construct an example for this hypersurface family.

Example 3. Let $\gamma(\nu)$ be a curve which is parametrized by

$$
\gamma(\nu)=\left(\nu, \sin v, \cos v, \nu^{3}\right) .
$$

From (4) and (5), it is easy to obtain that

$$
\left\{\begin{array}{l}
T(\nu)=\left(1, \cos v,-\sin v, 3 \nu^{2}\right), \\
N(\nu)=\frac{1}{\sqrt{1+36 \nu^{2}}}(0,-\sin v,-\cos v, 6 v), \\
B_{1}(\nu)=\frac{1}{\sqrt{\left(1+36 \nu^{2}\right)\left(37+36 \nu^{2}\right)}}\left(0,36 v \sin v-\left(36 v^{2}+1\right) \cos v,\right. \\
\left.36 v \cos v+\left(36 v^{2}+1\right) \sin v, 6\right), \\
B_{2}(\nu)=\frac{1}{\sqrt{37+36 \nu^{2}}}(0,6(\cos v+v \sin v), 6(v \cos v-\sin v), 1)
\end{array}\right.
$$

and

$$
\kappa_{1}=\sqrt{1+36 \nu^{2}}, \quad \kappa_{2}=\frac{\sqrt{37+36 \nu^{2}}}{1+36 \nu^{2}}, \quad \kappa_{3}=\frac{6 v \sqrt{1+36 \nu^{2}}}{37+36 \nu^{2}} .
$$

Now, if we choose $\sigma_{0}=\frac{\pi}{2}, \mu_{0}=0, x(\nu, \mu, \sigma)=\nu^{3}\left(\sigma-\frac{\pi}{2}\right), y(\nu, \mu, \sigma)=\nu \mu^{2} \sin \sigma$, $z(\nu, \mu, \sigma)=\cos \sigma$ and $m(\nu, \mu, \sigma)=\nu \mu \sigma^{2}$, then (26) is a geodesic curve where $N B_{2}$ Smarandache curve $r_{\mathrm{NB}_{2}}$ of the curve (26) is isoparametric on the hypersurface

$$
\begin{aligned}
& \varphi_{N B_{2}}(\nu, \mu, \sigma)=\left(\nu^{3}\left(\sigma-\frac{\pi}{2}\right),\right. \\
& \frac{-\sin v}{\sqrt{1+36 \nu^{2}}}+\frac{6(\cos v+v \sin v)}{\sqrt{\left(37+36 \nu^{2}\right)}}+\nu^{3}\left(\sigma-\frac{\pi}{2}\right) \cos v-\frac{\nu \mu^{2} \sin \sigma \sin v}{\sqrt{1+36 \nu^{2}}} \\
&+\frac{\cos \sigma\left(36 v \sin v-\left(36 v^{2}+1\right) \cos v\right)}{\sqrt{\left(1+36 \nu^{2}\right)\left(37+36 \nu^{2}\right)}}+\frac{6(\cos v+v \sin v) \nu \mu \sigma^{2}}{\sqrt{\left(37+36 \nu^{2}\right)}}
\end{aligned}
$$




$$
\begin{aligned}
& \frac{-\cos v}{\sqrt{1+36 \nu^{2}}}+\frac{6(v \cos v-\sin v)}{\sqrt{\left(37+36 \nu^{2}\right)}}-\nu^{3}\left(\sigma-\frac{\pi}{2}\right) \sin v-\frac{\nu \mu^{2} \sin \sigma \cos v}{\sqrt{1+36 \nu^{2}}} \\
& +\frac{\cos \sigma\left(36 v \cos v+\left(36 v^{2}+1\right) \sin v\right)}{\sqrt{\left(1+36 \nu^{2}\right)\left(37+36 \nu^{2}\right)}}+\frac{6(v \cos v-\sin v) \nu \mu \sigma^{2}}{\sqrt{\left(37+36 \nu^{2}\right)}}, \\
& \frac{6 v}{\sqrt{1+36 \nu^{2}}}+\frac{1}{\sqrt{\left(37+36 \nu^{2}\right)}}+3 v^{5}\left(\sigma-\frac{\pi}{2}\right)+\frac{6 \nu^{2} \mu^{2} \sin \sigma}{\sqrt{1+36 \nu^{2}}} \\
& \left.+\frac{6 \cos \sigma}{\sqrt{\left(1+36 \nu^{2}\right)\left(37+36 \nu^{2}\right)}}+\frac{\nu \mu \sigma^{2}}{\sqrt{\left(37+36 \nu^{2}\right)}}\right) .
\end{aligned}
$$

in $G_{4}$.

Different projections from four-space to three-spaces of the hypersurface 29) for $\sigma=\pi / 2$ can be seen in the Fig 1 :

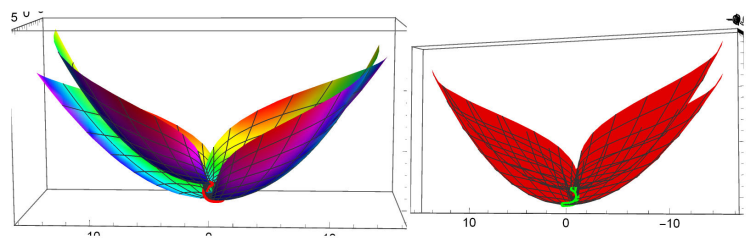

(a)

(b)

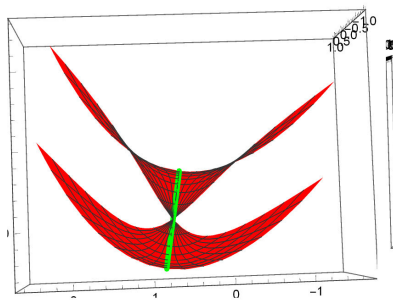

(c)

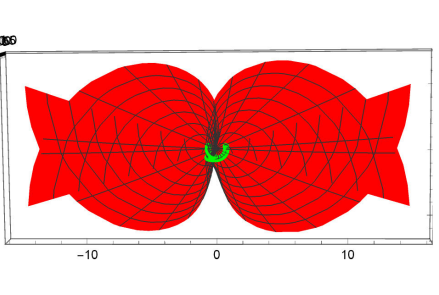

(d)

FiguRE 1. Projections of hypersurface family with parameter $N B_{2}$-Smarandache curve into $x_{2} x_{3} x_{4}, x_{1} x_{3} x_{4}, x_{1} x_{2} x_{4}$ and $x_{1} x_{2} x_{3}$ spaces in (a), (b), (c) and (d), respectively.

From now on, we'll give the parametric hypersurfaces given by different Smarandache curves of curve $\gamma(\nu)$ and their normal vector fields. Also, we'll state the theorems which give us the conditions for which $\gamma(\nu)$ is a geodesic curve where 
Smarandache curves of the curve $\gamma(\nu)$ is isoparametric on these hypersurfaces. One can prove these theorems and investigate the conditions for different types of marching-scale functions with the similar methods given in the above case.

CASE 3.

Here, by taking the $T B_{1}$-Smarandache curve of $\gamma(\nu)$ instead of the curve $\gamma(\nu)$ in (9), let us define a parametric hypersurface $\varphi_{T B_{1}}(\nu, \mu, \sigma)$ which is given with the aid of the $T B_{1}$-Smarandache curve of $\gamma(\nu)$ and the Frenet vectors of the curve $\gamma(\nu)$ as follows

$$
\varphi_{T B_{1}}(\nu, \mu, \sigma)=r_{T B_{1}}(\nu)+\left[\begin{array}{c}
x(\nu, \mu, \sigma) T(\nu)+y(\nu, \mu, \sigma) N(\nu) \\
+z(\nu, \mu, \sigma) B_{1}(\nu)+m(\nu, \mu, \sigma) B_{2}(\nu)
\end{array}\right] .
$$

If $T B_{1}$-Smarandache curve of the curve $\gamma(\nu)$ is a isoparametric curve on a hypersurface $\varphi_{T B_{1}}(\nu, \mu, \sigma)$ in $G_{4}$ for $\mu=\mu_{0}$ and $\sigma=\sigma_{0}$, then from (8), the normal of this hypersurface is

$$
\begin{aligned}
\eta_{T B_{1}}\left(\nu, \mu_{0}, \sigma_{0}\right) & =\left(\eta_{T B_{1}}\right)_{1}\left(\nu, \mu_{0}, \sigma_{0}\right) T(\nu)+\left(\eta_{T B_{1}}\right)_{2}\left(\nu, \mu_{0}, \sigma_{0}\right) N(\nu) \\
& +\left(\eta_{T B_{1}}\right)_{3}\left(\nu, \mu_{0}, \sigma_{0}\right) B_{1}(\nu)+\left(\eta_{T B_{1}}\right)_{4}\left(\nu, \mu_{0}, \sigma_{0}\right) B_{2}(\nu),
\end{aligned}
$$

where

$$
\begin{aligned}
\left(\eta_{T B_{1}}\right)_{1}\left(\nu, \mu_{0}, \sigma_{0}\right) & =0 \\
\left(\eta_{T B_{1}}\right)_{2}\left(\nu, \mu_{0}, \sigma_{0}\right) & =\kappa_{3}\left(\left(x_{\sigma}\right)_{0}\left(z_{\mu}\right)_{0}-\left(z_{\sigma}\right)_{0}\left(x_{\mu}\right)_{0}\right) \\
\left(\eta_{T B_{1}}\right)_{3}\left(\nu, \mu_{0}, \sigma_{0}\right) & =\left(\kappa_{1}-\kappa_{2}\right)\left(\left(m_{\mu}\right)_{0}\left(x_{\sigma}\right)_{0}-\left(x_{\mu}\right)_{0}\left(m_{\sigma}\right)_{0}\right) \\
& +\kappa_{3}\left(\left(x_{\mu}\right)_{0}\left(y_{\sigma}\right)_{0}-\left(y_{\mu}\right)_{0}\left(x_{\sigma}\right)_{0}\right) \\
\left(\eta_{T B_{1}}\right)_{4}\left(\nu, \mu_{0}, \sigma_{0}\right) & =\left(\kappa_{1}-\kappa_{2}\right)\left(\left(x_{\mu}\right)_{0}\left(z_{\sigma}\right)_{0}-\left(z_{\mu}\right)_{0}\left(x_{\sigma}\right)_{0}\right) .
\end{aligned}
$$

Thus,

Theorem 4. $\gamma(\nu)$ is a geodesic curve where $T B_{1}$-Smarandache curve $r_{T B_{1}}$ of the curve $\gamma(\nu)$ is isoparametric on the hypersurface $\varphi_{T B_{1}}(\nu, \mu, \sigma)$ in $G_{4}$ if the conditions

$$
\left\{\begin{array}{l}
x\left(\nu, \mu_{0}, \sigma_{0}\right)=y\left(\nu, \mu_{0}, \sigma_{0}\right)=z\left(\nu, \mu_{0}, \sigma_{0}\right)=m\left(\nu, \mu_{0}, \sigma_{0}\right)=0, \\
\kappa_{1}=\kappa_{2}, \kappa_{3} \neq 0, \\
\left(y_{\mu}\right)_{0}\left(x_{\sigma}\right)_{0}=\left(x_{\mu}\right)_{0}\left(y_{\sigma}\right)_{0}, \quad\left(z_{\mu}\right)_{0}\left(x_{\sigma}\right)_{0} \neq\left(x_{\mu}\right)_{0}\left(z_{\sigma}\right)_{0}
\end{array}\right.
$$

are satisfied. Here, $\nu \in\left[L_{1}, L_{2}\right], \mu_{0} \in\left[T_{1}, T_{2}\right], \sigma_{0} \in\left[M_{1}, M_{2}\right]$.

\section{CASE 4.}

Here, by taking the $T B_{2}$-Smarandache curve of $\gamma(\nu)$ instead of the curve $\gamma(\nu)$ in (9), let us define a parametric hypersurface $\varphi_{T B_{2}}(\nu, \mu, \sigma)$ which is given with the aid of the $T B_{2}$-Smarandache curve of $\gamma(\nu)$ and the Frenet vectors of the curve $\gamma(\nu)$ as follows

$$
\varphi_{T B_{2}}(\nu, \mu, \sigma)=r_{T B_{2}}(\nu)+\left[\begin{array}{c}
x(\nu, \mu, \sigma) T(\nu)+y(\nu, \mu, \sigma) N(\nu) \\
+z(\nu, \mu, \sigma) B_{1}(\nu)+m(\nu, \mu, \sigma) B_{2}(\nu)
\end{array}\right] .
$$


If $T B_{2}$-Smarandache curve of the curve $\gamma(\nu)$ is a isoparametric curve on a hypersurface $\varphi_{T B_{2}}(\nu, \mu, \sigma)$ in $G_{4}$ for $\mu=\mu_{0}$ and $\sigma=\sigma_{0}$, then from (8), the normal of this hypersurface is

$$
\begin{aligned}
\eta_{T B_{2}}\left(\nu, \mu_{0}, \sigma_{0}\right) & =\left(\eta_{T B_{2}}\right)_{1}\left(\nu, \mu_{0}, \sigma_{0}\right) T(\nu)+\left(\eta_{T B_{2}}\right)_{2}\left(\nu, \mu_{0}, \sigma_{0}\right) N(\nu) \\
& +\left(\eta_{T B_{2}}\right)_{3}\left(\nu, \mu_{0}, \sigma_{0}\right) B_{1}(\nu)+\left(\eta_{T B_{2}}\right)_{4}\left(\nu, \mu_{0}, \sigma_{0}\right) B_{2}(\nu),
\end{aligned}
$$

where

$$
\begin{aligned}
& \left(\eta_{T B_{2}}\right)_{1}\left(\nu, \mu_{0}, \sigma_{0}\right)=0, \\
& \left(\eta_{T B_{2}}\right)_{2}\left(\nu, \mu_{0}, \sigma_{0}\right)=\kappa_{3}\left(\left(x_{\sigma}\right)_{0}\left(m_{\mu}\right)_{0}-\left(m_{\sigma}\right)_{0}\left(x_{\mu}\right)_{0}\right), \\
& \left(\eta_{T B_{2}}\right)_{3}\left(\nu, \mu_{0}, \sigma_{0}\right)=\kappa_{1}\left(\left(m_{\mu}\right)_{0}\left(x_{\sigma}\right)_{0}-\left(x_{\mu}\right)_{0}\left(m_{\sigma}\right)_{0}\right), \\
& \left(\eta_{T B_{2}}\right)_{4}\left(\nu, \mu_{0}, \sigma_{0}\right)=\kappa_{1}\left(\left(x_{\mu}\right)_{0}\left(z_{\sigma}\right)_{0}-\left(z_{\mu}\right)_{0}\left(x_{\sigma}\right)_{0}\right)+\kappa_{3}\left(\left(y_{\sigma}\right)_{0}\left(x_{\mu}\right)_{0}-\left(x_{\sigma}\right)_{0}\left(y_{\mu}\right)_{0}\right) .
\end{aligned}
$$

For the curve $\gamma(\nu)$ to be a geodesic where $T B_{2}$-Smarandache curve $r_{T B_{2}}$ of the curve $\gamma(\nu)$ is isoparametric on the hypersurface $\varphi_{T B_{1}}(\nu, \mu, \sigma)$ in $G_{4}$, the following conditions must hold:

$$
\left\{\begin{array}{l}
x\left(\nu, \mu_{0}, \sigma_{0}\right)=y\left(\nu, \mu_{0}, \sigma_{0}\right)=z\left(\nu, \mu_{0}, \sigma_{0}\right)=m\left(\nu, \mu_{0}, \sigma_{0}\right)=0, \\
\kappa_{1}=0, \kappa_{3} \neq 0, \\
\left(y_{\mu}\right)_{0}\left(x_{\sigma}\right)_{0}=\left(x_{\mu}\right)_{0}\left(y_{\sigma}\right)_{0},\left(m_{\mu}\right)_{0}\left(x_{\sigma}\right)_{0} \neq\left(x_{\mu}\right)_{0}\left(m_{\sigma}\right)_{0}
\end{array},\right.
$$

where $\nu \in\left[L_{1}, L_{2}\right], \mu_{0} \in\left[T_{1}, T_{2}\right], \sigma_{0} \in\left[M_{1}, M_{2}\right]$. But, from our assumption that $\kappa_{1}(v) \neq 0$, we have a contradiction. So, we have

Theorem 5. $\gamma(\nu)$ is not a geodesic curve where $T B_{2}$-Smarandache curve $r_{T B_{2}}$ of the curve $\gamma(\nu)$ is isoparametric on the hypersurface $\varphi_{T B_{2}}(\nu, \mu, \sigma)$ in $G_{4}$.

CASE 5.

Here, by taking the $N B_{1}$-Smarandache curve of $\gamma(\nu)$ instead of the curve $\gamma(\nu)$ in $\left(9 p\right.$, let us define a parametric hypersurface $\varphi_{N B_{1}}(\nu, \mu, \sigma)$ which is given with the aid of the $N B_{1}$-Smarandache curve of $\gamma(\nu)$ and the Frenet vectors of the curve $\gamma(\nu)$ as follows

$$
\varphi_{N B_{1}}(\nu, \mu, \sigma)=r_{N B_{1}}(\nu)+\left[\begin{array}{c}
x(\nu, \mu, \sigma) T(\nu)+y(\nu, \mu, \sigma) N(\nu) \\
+z(\nu, \mu, \sigma) B_{1}(\nu)+m(\nu, \mu, \sigma) B_{2}(\nu)
\end{array}\right] .
$$

If $N B_{1}$-Smarandache curve of the curve $\gamma(\nu)$ is a isoparametric curve on a hypersurface $\varphi_{N B_{1}}(\nu, \mu, \sigma)$ in $G_{4}$ for $\mu=\mu_{0}$ and $\sigma=\sigma_{0}$, then from (8), the normal of this hypersurface is

$$
\begin{aligned}
\eta_{N B_{1}}\left(\nu, \mu_{0}, \sigma_{0}\right) & =\left(\eta_{N B_{1}}\right)_{1}\left(\nu, \mu_{0}, \sigma_{0}\right) T(\nu)+\left(\eta_{N B_{1}}\right)_{2}\left(\nu, \mu_{0}, \sigma_{0}\right) N(\nu) \\
& +\left(\eta_{N B_{1}}\right)_{3}\left(\nu, \mu_{0}, \sigma_{0}\right) B_{1}(\nu)+\left(\eta_{N B_{1}}\right)_{4}\left(\nu, \mu_{0}, \sigma_{0}\right) B_{2}(\nu)
\end{aligned}
$$


where

$$
\begin{aligned}
\left(\eta_{N B_{1}}\right)_{1}\left(\nu, \mu_{0}, \sigma_{0}\right)= & 0, \\
\left(\eta_{N B_{1}}\right)_{2}\left(\nu, \mu_{0}, \sigma_{0}\right)= & \frac{\kappa_{2}}{\sqrt{2}}\left(\left(x_{\mu}\right)_{0}\left(m_{\sigma}\right)_{0}-\left(m_{\mu}\right)_{0}\left(x_{\sigma}\right)_{0}\right) \\
& -\frac{\kappa_{3}}{\sqrt{2}}\left(\left(x_{\mu}\right)_{0}\left(z_{\sigma}\right)_{0}-\left(z_{\mu}\right)_{0}\left(x_{\sigma}\right)_{0}\right), \\
\left(\eta_{N B_{1}}\right)_{3}\left(\nu, \mu_{0}, \sigma_{0}\right)= & \frac{\kappa_{2}}{\sqrt{2}}\left(\left(m_{\sigma}\right)_{0}\left(x_{\mu}\right)_{0}-\left(x_{\sigma}\right)_{0}\left(m_{\mu}\right)_{0}\right) \\
& +\frac{\kappa_{3}}{\sqrt{2}}\left(\left(x_{\mu}\right)_{0}\left(y_{\sigma}\right)_{0}-\left(y_{\mu}\right)_{0}\left(x_{\sigma}\right)_{0}\right), \\
\left(\eta_{N B_{1}}\right)_{4}\left(\nu, \mu_{0}, \sigma_{0}\right)= & \frac{\kappa_{2}}{\sqrt{2}}\left(\left(x_{\sigma}\right)_{0}\left(z_{\mu}\right)_{0}-\left(z_{\sigma}\right)_{0}\left(x_{\mu}\right)_{0}\right) \\
& +\frac{\kappa_{2}}{\sqrt{2}}\left(\left(x_{\sigma}\right)_{0}\left(y_{\mu}\right)_{0}-\left(y_{\sigma}\right)_{0}\left(x_{\mu}\right)_{0}\right) . \\
&
\end{aligned}
$$

Hence,

Theorem 6. $\gamma(\nu)$ is a geodesic curve where $N B_{1}$-Smarandache curve $r_{N B_{1}}$ of the curve $\gamma(\nu)$ is isoparametric on the hypersurface $\varphi_{N B_{1}}(\nu, \mu, \sigma)$ in $G_{4}$ if the conditions

$$
\left\{\begin{array}{l}
x\left(\nu, \mu_{0}, \sigma_{0}\right)=y\left(\nu, \mu_{0}, \sigma_{0}\right)=z\left(\nu, \mu_{0}, \sigma_{0}\right)=m\left(\nu, \mu_{0}, \sigma_{0}\right)=0 \\
\left(\eta_{N B_{1}}\right)_{2}\left(\nu, \mu_{0}, \sigma_{0}\right) \neq 0, \quad\left(\eta_{N B_{1}}\right)_{3}\left(\nu, \mu_{0}, \sigma_{0}\right)=\left(\eta_{N B_{1}}\right)_{4}\left(\nu, \mu_{0}, \sigma_{0}\right)=0
\end{array}\right.
$$

are satisfied. Here, $\nu \in\left[L_{1}, L_{2}\right], \mu_{0} \in\left[T_{1}, T_{2}\right], \sigma_{0} \in\left[M_{1}, M_{2}\right]$.

CASE 6.

Here, by taking the $B_{1} B_{2}$-Smarandache curve of $\gamma(\nu)$ instead of the curve $\gamma(\nu)$ in (9), let us define a parametric hypersurface $\varphi_{B_{1} B_{2}}(\nu, \mu, \sigma)$ which is given with the aid of the $B_{1} B_{2}$-Smarandache curve of $\gamma(\nu)$ and the Frenet vectors of the curve $\gamma(\nu)$ as follows

$$
\varphi_{B_{1} B_{2}}(\nu, \mu, \sigma)=r_{B_{1} B_{2}}(\nu)+\left[\begin{array}{c}
x(\nu, \mu, \sigma) T(\nu)+y(\nu, \mu, \sigma) N(\nu) \\
+z(\nu, \mu, \sigma) B_{1}(\nu)+m(\nu, \mu, \sigma) B_{2}(\nu)
\end{array}\right] .
$$

If $B_{1} B_{2}$-Smarandache curve of the curve $\gamma(\nu)$ is a isoparametric curve on a hypersurface $\varphi_{B_{1} B_{2}}(\nu, \mu, \sigma)$ in $G_{4}$ for $\mu=\mu_{0}$ and $\sigma=\sigma_{0}$, then from (8), the normal of this hypersurface is

$$
\begin{aligned}
\eta_{B_{1} B_{2}}\left(\nu, \mu_{0}, \sigma_{0}\right) & =\left(\eta_{B_{1} B_{2}}\right)_{1}\left(\nu, \mu_{0}, \sigma_{0}\right) T(\nu)+\left(\eta_{B_{1} B_{2}}\right)_{2}\left(\nu, \mu_{0}, \sigma_{0}\right) N(\nu) \\
& +\left(\eta_{B_{1} B_{2}}\right)_{3}\left(\nu, \mu_{0}, \sigma_{0}\right) B_{1}(\nu)+\left(\eta_{B_{1} B_{2}}\right)_{4}\left(\nu, \mu_{0}, \sigma_{0}\right) B_{2}(\nu),
\end{aligned}
$$

where

$$
\begin{aligned}
& \left(\eta_{B_{1} B_{2}}\right)_{1}\left(\nu, \mu_{0}, \sigma_{0}\right)=0 \\
& \left(\eta_{B_{1} B_{2}}\right)_{2}\left(\nu, \mu_{0}, \sigma_{0}\right)=\frac{\kappa_{3}}{\sqrt{2}}\left(\left(x_{\sigma}\right)_{0}\left(\left(m_{\mu}\right)_{0}+\left(z_{\mu}\right)_{0}\right)-\left(x_{\mu}\right)_{0}\left(\left(m_{\sigma}\right)_{0}+\left(z_{\sigma}\right)_{0}\right)\right), \\
& \left(\eta_{B_{1} B_{2}}\right)_{3}\left(\nu, \mu_{0}, \sigma_{0}\right)=\frac{\kappa_{2}}{\sqrt{2}}\left(\left(x_{\mu}\right)_{0}\left(m_{\sigma}\right)_{0}-\left(m_{\mu}\right)_{0}\left(x_{\sigma}\right)_{0}\right)+\frac{\kappa_{3}}{\sqrt{2}}\left(\left(x_{\mu}\right)_{0}\left(y_{\sigma}\right)_{0}-\left(y_{\mu}\right)_{0}\left(x_{\sigma}\right)_{0}\right), \\
& \left(\eta_{B_{1} B_{2}}\right)_{4}\left(\nu, \mu_{0}, \sigma_{0}\right)=\frac{\kappa_{2}}{\sqrt{2}}\left(\left(z_{\mu}\right)_{0}\left(x_{\sigma}\right)_{0}-\left(x_{\mu}\right)_{0}\left(z_{\sigma}\right)_{0}\right)-\frac{\kappa_{3}}{\sqrt{2}}\left(\left(y_{\mu}\right)_{0}\left(x_{\sigma}\right)_{0}-\left(x_{\mu}\right)_{0}\left(y_{\sigma}\right)_{0}\right) .
\end{aligned}
$$

So, we can state the following Theorem: 
Theorem 7. $\gamma(\nu)$ is a geodesic curve where $B_{1} B_{2}$-Smarandache curve $r_{B_{1} B_{2}}$ of the curve $\gamma(\nu)$ is isoparametric on the hypersurface $\varphi_{B_{1} B_{2}}(\nu, \mu, \sigma)$ in $G_{4}$ if the conditions

$$
\left\{\begin{array}{l}
x\left(\nu, \mu_{0}, \sigma_{0}\right)=y\left(\nu, \mu_{0}, \sigma_{0}\right)=z\left(\nu, \mu_{0}, \sigma_{0}\right)=m\left(\nu, \mu_{0}, \sigma_{0}\right)=0 \\
\kappa_{3} \neq 0 \\
\left(x_{\mu}\right)_{0}\left(\left(m_{\sigma}\right)_{0}+\left(z_{\sigma}\right)_{0}\right) \neq\left(x_{\sigma}\right)_{0}\left(\left(m_{\mu}\right)_{0}+\left(z_{\mu}\right)_{0}\right) \\
\left(\eta_{B_{1} B_{2}}\right)_{3}\left(\nu, \mu_{0}, \sigma_{0}\right)=\left(\eta_{B_{1} B_{2}}\right)_{4}\left(\nu, \mu_{0}, \sigma_{0}\right)=0
\end{array}\right.
$$

are satisfied. Here, $\nu \in\left[L_{1}, L_{2}\right], \mu_{0} \in\left[T_{1}, T_{2}\right], \sigma_{0} \in\left[M_{1}, M_{2}\right]$.

CASE 7.

Here, by taking the $T N B_{1}$-Smarandache curve of $\gamma(\nu)$ instead of the curve $\gamma(\nu)$ in (9), let us define a parametric hypersurface $\varphi_{T N B_{1}}(\nu, \mu, \sigma)$ which is given with the aid of the $T N B_{1}$-Smarandache curve of $\gamma(\nu)$ and the Frenet vectors of the curve $\gamma(\nu)$ as follows

$$
\varphi_{T N B_{1}}(\nu, \mu, \sigma)=r_{T N B_{1}}(\nu)+\left[\begin{array}{c}
x(\nu, \mu, \sigma) T(\nu)+y(\nu, \mu, \sigma) N(\nu) \\
+z(\nu, \mu, \sigma) B_{1}(\nu)+m(\nu, \mu, \sigma) B_{2}(\nu)
\end{array}\right] .
$$

If $T N B_{1}$-Smarandache curve of the curve $\gamma(\nu)$ is a isoparametric curve on a hypersurface $\varphi_{T N B_{1}}(\nu, \mu, \sigma)$ in $G_{4}$ for $\mu=\mu_{0}$ and $\sigma=\sigma_{0}$, then from (8), the normal of this hypersurface is

$$
\begin{aligned}
\eta_{T N B_{1}}\left(\nu, \mu_{0}, \sigma_{0}\right) & =\left(\eta_{T N B_{1}}\right)_{1}\left(\nu, \mu_{0}, \sigma_{0}\right) T(\nu)+\left(\eta_{T N B_{1}}\right)_{2}\left(\nu, \mu_{0}, \sigma_{0}\right) N(\nu) \\
& +\left(\eta_{T N B_{1}}\right)_{3}\left(\nu, \mu_{0}, \sigma_{0}\right) B_{1}(\nu)+\left(\eta_{T N B_{1}}\right)_{4}\left(\nu, \mu_{0}, \sigma_{0}\right) B_{2}(\nu),
\end{aligned}
$$

where

$$
\begin{aligned}
\left(\eta_{T N B_{1}}\right)_{1}\left(\nu, \mu_{0}, \sigma_{0}\right) & =0 \\
\left(\eta_{T N B_{1}}\right)_{2}\left(\nu, \mu_{0}, \sigma_{0}\right) & =\kappa_{3}\left(\left(z_{\mu}\right)_{0}\left(x_{\sigma}\right)_{0}-\left(x_{\mu}\right)_{0}\left(z_{\sigma}\right)_{0}\right)-\kappa_{2}\left(\left(m_{\mu}\right)_{0}\left(x_{\sigma}\right)_{0}-\left(x_{\mu}\right)_{0}\left(m_{\sigma}\right)_{0}\right), \\
\left(\eta_{T N B_{1}}\right)_{3}\left(\nu, \mu_{0}, \sigma_{0}\right) & =\left(\kappa_{2}-\kappa_{1}\right)\left(\left(x_{\mu}\right)_{0}\left(m_{\sigma}\right)_{0}-\left(m_{\mu}\right)_{0}\left(x_{\sigma}\right)_{0}\right) \\
& +\kappa_{3}\left(\left(x_{\mu}\right)_{0}\left(y_{\sigma}\right)_{0}-\left(y_{\mu}\right)_{0}\left(x_{\sigma}\right)_{0}\right) \\
\left(\eta_{T N B_{1}}\right)_{4}\left(\nu, \mu_{0}, \sigma_{0}\right) & =\left(\kappa_{1}-\kappa_{2}\right)\left(\left(x_{\mu}\right)_{0}\left(z_{\sigma}\right)_{0}-\left(z_{\mu}\right)_{0}\left(x_{\sigma}\right)_{0}\right) \\
& +\kappa_{2}\left(\left(y_{\mu}\right)_{0}\left(x_{\sigma}\right)_{0}-\left(x_{\mu}\right)_{0}\left(y_{\sigma}\right)_{0}\right) .
\end{aligned}
$$

Hence,

Theorem 8. $\gamma(\nu)$ is a geodesic curve where $T N B_{1}$-Smarandache curve $r_{T N B_{1}}$ of the curve $\gamma(\nu)$ is isoparametric on the hypersurface $\varphi_{T N B_{1}}(\nu, \mu, \sigma)$ in $G_{4}$ if the conditions

$$
\left\{\begin{array}{l}
x\left(\nu, \mu_{0}, \sigma_{0}\right)=y\left(\nu, \mu_{0}, \sigma_{0}\right)=z\left(\nu, \mu_{0}, \sigma_{0}\right)=m\left(\nu, \mu_{0}, \sigma_{0}\right)=0 \\
\left(\eta_{T N B_{1}}\right)_{2}\left(\nu, \mu_{0}, \sigma_{0}\right) \neq 0,\left(\eta_{T N B_{1}}\right)_{3}\left(\nu, \mu_{0}, \sigma_{0}\right)=\left(\eta_{T N B_{1}}\right)_{4}\left(\nu, \mu_{0}, \sigma_{0}\right)=0
\end{array}\right.
$$

are satisfied. Here, $\nu \in\left[L_{1}, L_{2}\right], \mu_{0} \in\left[T_{1}, T_{2}\right], \sigma_{0} \in\left[M_{1}, M_{2}\right]$. 


\section{CASE 8.}

Here, by taking the $T N B_{2}$-Smarandache curve of $\gamma(\nu)$ instead of the curve $\gamma(\nu)$ in (9), let us define a parametric hypersurface $\varphi_{T N B_{2}}(\nu, \mu, \sigma)$ which is given with the aid of the $T \mathrm{NB}_{2}$-Smarandache curve of $\gamma(\nu)$ and the Frenet vectors of the curve $\gamma(\nu)$ as follows

$$
\varphi_{T N B_{2}}(\nu, \mu, \sigma)=r_{T N B_{2}}(\nu)+\left[\begin{array}{c}
x(\nu, \mu, \sigma) T(\nu)+y(\nu, \mu, \sigma) N(\nu) \\
+z(\nu, \mu, \sigma) B_{1}(\nu)+m(\nu, \mu, \sigma) B_{2}(\nu)
\end{array}\right] .
$$

If $T N B_{2}$-Smarandache curve of the curve $\gamma(\nu)$ is a isoparametric curve on a hypersurface $\varphi_{T N B_{2}}(\nu, \mu, \sigma)$ in $G_{4}$ for $\mu=\mu_{0}$ and $\sigma=\sigma_{0}$, then from (8), the normal of this hypersurface is

$$
\begin{aligned}
\eta_{T N B_{2}}\left(\nu, \mu_{0}, \sigma_{0}\right) & =\left(\eta_{T N B_{2}}\right)_{1}\left(\nu, \mu_{0}, \sigma_{0}\right) T(\nu)+\left(\eta_{T N B_{2}}\right)_{2}\left(\nu, \mu_{0}, \sigma_{0}\right) N(\nu) \\
& +\left(\eta_{T N B_{2}}\right)_{3}\left(\nu, \mu_{0}, \sigma_{0}\right) B_{1}(\nu)+\left(\eta_{T N B_{2}}\right)_{4}\left(\nu, \mu_{0}, \sigma_{0}\right) B_{2}(\nu),
\end{aligned}
$$

where

$$
\begin{aligned}
\left(\eta_{T N B_{2}}\right)_{1}\left(\nu, \mu_{0}, \sigma_{0}\right) & =0, \\
\left(\eta_{T N B_{2}}\right)_{2}\left(\nu, \mu_{0}, \sigma_{0}\right) & =\left(\kappa_{2}-\kappa_{3}\right)\left(\left(x_{\mu}\right)_{0}\left(m_{\sigma}\right)_{0}-\left(m_{\mu}\right)_{0}\left(x_{\sigma}\right)_{0}\right), \\
\left(\eta_{T N B_{2}}\right)_{3}\left(\nu, \mu_{0}, \sigma_{0}\right) & =-\kappa_{1}\left(\left(x_{\mu}\right)_{0}\left(m_{\sigma}\right)_{0}-\left(m_{\mu}\right)_{0}\left(x_{\sigma}\right)_{0}\right) \\
\left(\eta_{T N B_{2}}\right)_{4}\left(\nu, \mu_{0}, \sigma_{0}\right) & =\kappa_{1}\left(\left(x_{\mu}\right)_{0}\left(z_{\sigma}\right)_{0}-\left(z_{\mu}\right)_{0}\left(x_{\sigma}\right)_{0}\right) \\
& +\left(\kappa_{3}-\kappa_{2}\right)\left(\left(x_{\mu}\right)_{0}\left(y_{\sigma}\right)_{0}-\left(y_{\mu}\right)_{0}\left(x_{\sigma}\right)_{0}\right) .
\end{aligned}
$$

For the curve $\gamma(\nu)$ to be a geodesic where $T N B_{2}$-Smarandache curve $r_{T N B_{2}}$ of the curve $\gamma(\nu)$ is isoparametric on the hypersurface $\varphi_{T N B_{2}}(\nu, \mu, \sigma)$ in $G_{4}$, the following conditions must hold:

$$
\left\{\begin{array}{l}
x\left(\nu, \mu_{0}, \sigma_{0}\right)=y\left(\nu, \mu_{0}, \sigma_{0}\right)=z\left(\nu, \mu_{0}, \sigma_{0}\right)=m\left(\nu, \mu_{0}, \sigma_{0}\right)=0, \\
\kappa_{1}=0, \kappa_{2} \neq \kappa_{3}, \\
\left(x_{\mu}\right)_{0}\left(y_{\sigma}\right)_{0}=\left(y_{\mu}\right)_{0}\left(x_{\sigma}\right)_{0},\left(x_{\mu}\right)_{0}\left(m_{\sigma}\right)_{0} \neq\left(m_{\mu}\right)_{0}\left(x_{\sigma}\right)_{0}
\end{array},\right.
$$

where $\nu \in\left[L_{1}, L_{2}\right], \mu_{0} \in\left[T_{1}, T_{2}\right], \sigma_{0} \in\left[M_{1}, M_{2}\right]$. Since $\kappa_{1}(v) \neq 0$, we have

Theorem 9. $\gamma(\nu)$ is not a geodesic curve where $T N B_{2}$-Smarandache curve $r_{T N B_{2}}$ of the curve $\gamma(\nu)$ is isoparametric on the hypersurface $\varphi_{T N B_{2}}(\nu, \mu, \sigma)$ in $G_{4}$.

\section{CASE 9.}

Here, by taking the $T B_{1} B_{2}$-Smarandache curve of $\gamma(\nu)$ instead of the curve $\gamma(\nu)$ in $(9)$, let us define a parametric hypersurface $\varphi_{T B_{1} B_{2}}(\nu, \mu, \sigma)$ which is given with the aid of the $T B_{1} B_{2}$-Smarandache curve of $\gamma(\nu)$ and the Frenet vectors of the curve $\gamma(\nu)$ as follows

$$
\varphi_{T B_{1} B_{2}}(\nu, \mu, \sigma)=r_{T B_{1} B_{2}}(\nu)+\left[\begin{array}{c}
x(\nu, \mu, \sigma) T(\nu)+y(\nu, \mu, \sigma) N(\nu) \\
+z(\nu, \mu, \sigma) B_{1}(\nu)+m(\nu, \mu, \sigma) B_{2}(\nu)
\end{array}\right] .
$$

If $T B_{1} B_{2}$-Smarandache curve of the curve $\gamma(\nu)$ is a isoparametric curve on a hypersurface $\varphi_{T B_{1} B_{2}}(\nu, \mu, \sigma)$ in $G_{4}$ for $\mu=\mu_{0}$ and $\sigma=\sigma_{0}$, then from (8), the 
normal of this hypersurface is

$$
\begin{aligned}
\eta_{T B_{1} B_{2}}\left(\nu, \mu_{0}, \sigma_{0}\right) & =\left(\eta_{T B_{1} B_{2}}\right)_{1}\left(\nu, \mu_{0}, \sigma_{0}\right) T(\nu)+\left(\eta_{T B_{1} B_{2}}\right)_{2}\left(\nu, \mu_{0}, \sigma_{0}\right) N(\nu) \\
& +\left(\eta_{T B_{1} B_{2}}\right)_{3}\left(\nu, \mu_{0}, \sigma_{0}\right) B_{1}(\nu)+\left(\eta_{T B_{1} B_{2}}\right)_{4}\left(\nu, \mu_{0}, \sigma_{0}\right) B_{2}(\nu),
\end{aligned}
$$

where

$$
\begin{aligned}
\left(\eta_{T B_{1} B_{2}}\right)_{1}\left(\nu, \mu_{0}, \sigma_{0}\right) & =0 \\
\left(\eta_{T B_{1} B_{2}}\right)_{2}\left(\nu, \mu_{0}, \sigma_{0}\right) & =\kappa_{3}\left(\left(x_{\sigma}\right)_{0}\left(\left(m_{\mu}\right)_{0}+\left(z_{\mu}\right)_{0}\right)-\left(x_{\mu}\right)_{0}\left(\left(m_{\sigma}\right)_{0}+\left(z_{\sigma}\right)_{0}\right)\right), \\
\left(\eta_{T B_{1} B_{2}}\right)_{3}\left(\nu, \mu_{0}, \sigma_{0}\right) & =\left(\kappa_{2}-\kappa_{1}\right)\left(\left(x_{\mu}\right)_{0}\left(m_{\sigma}\right)_{0}-\left(m_{\mu}\right)_{0}\left(x_{\sigma}\right)_{0}\right) \\
& +\kappa_{3}\left(\left(x_{\mu}\right)_{0}\left(y_{\sigma}\right)_{0}-\left(y_{\mu}\right)_{0}\left(x_{\sigma}\right)_{0}\right) \\
\left(\eta_{T B_{1} B_{2}}\right)_{4}\left(\nu, \mu_{0}, \sigma_{0}\right) & =\left(\kappa_{1}-\kappa_{2}\right)\left(\left(x_{\mu}\right)_{0}\left(z_{\sigma}\right)_{0}-\left(z_{\mu}\right)_{0}\left(x_{\sigma}\right)_{0}\right) \\
& +\kappa_{3}\left(\left(x_{\mu}\right)_{0}\left(y_{\sigma}\right)_{0}-\left(y_{\mu}\right)_{0}\left(x_{\sigma}\right)_{0}\right) .
\end{aligned}
$$

Thus,

Theorem 10. $\gamma(\nu)$ is a geodesic curve where $T B_{1} B_{2}$-Smarandache curve $r_{T B_{1} B_{2}}$ of the curve $\gamma(\nu)$ is isoparametric on the hypersurface $\varphi_{T B_{1} B_{2}}(\nu, \mu, \sigma)$ in $G_{4}$ if the conditions

$$
\left\{\begin{array}{l}
x\left(\nu, \mu_{0}, \sigma_{0}\right)=y\left(\nu, \mu_{0}, \sigma_{0}\right)=z\left(\nu, \mu_{0}, \sigma_{0}\right)=m\left(\nu, \mu_{0}, \sigma_{0}\right)=0, \\
\kappa_{3} \neq 0, \\
\left(\eta_{T B_{1} B_{2}}\right)_{3}\left(\nu, \mu_{0}, \sigma_{0}\right)=\left(\eta_{T B_{1} B_{2}}\right)_{4}\left(\nu, \mu_{0}, \sigma_{0}\right)=0 \\
\left(x_{\mu}\right)_{0}\left(\left(m_{\sigma}\right)_{0}+\left(z_{\sigma}\right)_{0}\right) \neq\left(x_{\sigma}\right)_{0}\left(\left(m_{\mu}\right)_{0}+\left(z_{\mu}\right)_{0}\right)
\end{array}\right.
$$

are satisfied. Here, $\nu \in\left[L_{1}, L_{2}\right], \mu_{0} \in\left[T_{1}, T_{2}\right], \sigma_{0} \in\left[M_{1}, M_{2}\right]$.

\section{CASE 10.}

Here, by taking the $N B_{1} B_{2}$-Smarandache curve of $\gamma(\nu)$ instead of the curve $\gamma(\nu)$ in (9), let us define a parametric hypersurface $\varphi_{N B_{1} B_{2}}(\nu, \mu, \sigma)$ which is given with the aid of the $N B_{1} B_{2}$-Smarandache curve of $\gamma(\nu)$ and the Frenet vectors of the curve $\gamma(\nu)$ as follows

$$
\varphi_{N B_{1} B_{2}}(\nu, \mu, \sigma)=r_{N B_{1} B_{2}}(\nu)+\left[\begin{array}{c}
x(\nu, \mu, \sigma) T(\nu)+y(\nu, \mu, \sigma) N(\nu) \\
+z(\nu, \mu, \sigma) B_{1}(\nu)+m(\nu, \mu, \sigma) B_{2}(\nu)
\end{array}\right] .
$$

If $N B_{1} B_{2}$-Smarandache curve of the curve $\gamma(\nu)$ is a isoparametric curve on a hypersurface $\varphi_{N B_{1} B_{2}}(\nu, \mu, \sigma)$ in $G_{4}$ for $\mu=\mu_{0}$ and $\sigma=\sigma_{0}$, then from (8), the normal of this hypersurface is

$$
\begin{aligned}
\eta_{N B_{1} B_{2}}\left(\nu, \mu_{0}, \sigma_{0}\right) & =\left(\eta_{N B_{1} B_{2}}\right)_{1}\left(\nu, \mu_{0}, \sigma_{0}\right) T(\nu)+\left(\eta_{N B_{1} B_{2}}\right)_{2}\left(\nu, \mu_{0}, \sigma_{0}\right) N(\nu) \\
& +\left(\eta_{N B_{1} B_{2}}\right)_{3}\left(\nu, \mu_{0}, \sigma_{0}\right) B_{1}(\nu)+\left(\eta_{N B_{1} B_{2}}\right)_{4}\left(\nu, \mu_{0}, \sigma_{0}\right) B_{2}(\nu),
\end{aligned}
$$

where

$$
\begin{aligned}
& \left(\eta_{N B_{1} B_{2}}\right)_{1}\left(\nu, \mu_{0}, \sigma_{0}\right)=0 \\
& \left(\eta_{N B_{1} B_{2}}\right)_{2}\left(\nu, \mu_{0}, \sigma_{0}\right)=\left(\frac{\kappa_{2}-\kappa_{3}}{\sqrt{3}}\right)\left(\left(x_{\mu}\right)_{0}\left(m_{\sigma}\right)_{0}-\left(m_{\mu}\right)_{0}\left(x_{\sigma}\right)_{0}\right)
\end{aligned}
$$




$$
\begin{aligned}
& +\frac{\kappa_{3}}{\sqrt{3}}\left(\left(z_{\mu}\right)_{0}\left(x_{\sigma}\right)_{0}-\left(x_{\mu}\right)_{0}\left(z_{\sigma}\right)_{0}\right), \\
\left(\eta_{N B_{1} B_{2}}\right)_{3}\left(\nu, \mu_{0}, \sigma_{0}\right) & =\frac{\kappa_{2}}{\sqrt{3}}\left(\left(x_{\mu}\right)_{0}\left(m_{\sigma}\right)_{0}-\left(m_{\mu}\right)_{0}\left(x_{\sigma}\right)_{0}\right) \\
& +\frac{\kappa_{3}}{\sqrt{3}}\left(\left(x_{\mu}\right)_{0}\left(y_{\sigma}\right)_{0}-\left(y_{\mu}\right)_{0}\left(x_{\sigma}\right)_{0}\right), \\
\left(\eta_{N B_{1} B_{2}}\right)_{4}\left(\nu, \mu_{0}, \sigma_{0}\right) & =\frac{\kappa_{2}}{\sqrt{3}}\left(\left(z_{\mu}\right)_{0}\left(x_{\sigma}\right)_{0}-\left(x_{\mu}\right)_{0}\left(z_{\sigma}\right)_{0}\right) \\
& +\left(\frac{\kappa_{3}-\kappa_{2}}{\sqrt{3}}\right)\left(\left(x_{\mu}\right)_{0}\left(y_{\sigma}\right)_{0}-\left(y_{\mu}\right)_{0}\left(x_{\sigma}\right)_{0}\right) .
\end{aligned}
$$

So,

Theorem 11. $\gamma(\nu)$ is a geodesic curve where $N B_{1} B_{2}$-Smarandache curve $r_{N B_{1} B_{2}}$ of the curve $\gamma(\nu)$ is isoparametric on the hypersurface $\varphi_{N B_{1} B_{2}}(\nu, \mu, \sigma)$ in $G_{4}$ if the conditions

$$
\left\{\begin{array}{l}
x\left(\nu, \mu_{0}, \sigma_{0}\right)=y\left(\nu, \mu_{0}, \sigma_{0}\right)=z\left(\nu, \mu_{0}, \sigma_{0}\right)=m\left(\nu, \mu_{0}, \sigma_{0}\right)=0 \\
\left(\eta_{N B_{1} B_{2}}\right)_{2}\left(\nu, \mu_{0}, \sigma_{0}\right) \neq 0,\left(\eta_{N B_{1} B_{2}}\right)_{3}\left(\nu, \mu_{0}, \sigma_{0}\right)=\left(\eta_{N B_{1} B_{2}}\right)_{4}\left(\nu, \mu_{0}, \sigma_{0}\right)=0
\end{array}\right.
$$

are satisfied. Here, $\nu \in\left[L_{1}, L_{2}\right], \mu_{0} \in\left[T_{1}, T_{2}\right], \sigma_{0} \in\left[M_{1}, M_{2}\right]$.

\section{CASE 11.}

Here, by taking the $T N B_{1} B_{2}$-Smarandache curve of $\gamma(\nu)$ instead of the curve $\gamma(\nu)$ in $(9)$, let us define a parametric hypersurface $\varphi_{T N B_{1} B_{2}}(\nu, \mu, \sigma)$ which is given with the aid of the $T N B_{1} B_{2}$-Smarandache curve of $\gamma(\nu)$ and the Frenet vectors of the curve $\gamma(\nu)$ as follows

$$
\varphi_{T N B_{1} B_{2}}(\nu, \mu, \sigma)=r_{T N B_{1} B_{2}}(\nu)+\left[\begin{array}{c}
x(\nu, \mu, \sigma) T(\nu)+y(\nu, \mu, \sigma) N(\nu) \\
+z(\nu, \mu, \sigma) B_{1}(\nu)+m(\nu, \mu, \sigma) B_{2}(\nu)
\end{array}\right] .
$$

If $T N B_{1} B_{2}$-Smarandache curve of the curve $\gamma(\nu)$ is a isoparametric curve on a hypersurface $\varphi_{T N B_{1} B_{2}}(\nu, \mu, \sigma)$ in $G_{4}$ for $\mu=\mu_{0}$ and $\sigma=\sigma_{0}$, then from (8), the normal of this hypersurface is

$$
\begin{aligned}
\eta_{T N B_{1} B_{2}}\left(\nu, \mu_{0}, \sigma_{0}\right) & =\left(\eta_{T N B_{1} B_{2}}\right)_{1}\left(\nu, \mu_{0}, \sigma_{0}\right) T(\nu)+\left(\eta_{T N B_{1} B_{2}}\right)_{2}\left(\nu, \mu_{0}, \sigma_{0}\right) N(\nu) \\
& +\left(\eta_{T N B_{1} B_{2}}\right)_{3}\left(\nu, \mu_{0}, \sigma_{0}\right) B_{1}(\nu)+\left(\eta_{T N B_{1} B_{2}}\right)_{4}\left(\nu, \mu_{0}, \sigma_{0}\right) B_{2}(\nu),
\end{aligned}
$$

where

$$
\begin{aligned}
& \left(\eta_{T N B_{1} B_{2}}\right)_{1}\left(\nu, \mu_{0}, \sigma_{0}\right)=0, \\
& \left(\eta_{T N B_{1} B_{2}}\right)_{2}\left(\nu, \mu_{0}, \sigma_{0}\right)=\kappa_{2}\left(\left(m_{\sigma}\right)_{0}\left(x_{\mu}\right)_{0}-\left(x_{\sigma}\right)_{0}\left(m_{\mu}\right)_{0}\right) \\
& +\kappa_{3}\left(\left(\left(x_{\sigma}\right)_{0}\left(m_{\mu}\right)_{0}-\left(m_{\sigma}\right)_{0}\left(x_{\mu}\right)_{0}\right)-\left(\left(x_{\mu}\right)_{0}\left(z_{\sigma}\right)_{0}-\left(z_{\mu}\right)_{0}\left(x_{\sigma}\right)_{0}\right)\right), \\
& \left(\eta_{T N B_{1} B_{2}}\right)_{3}\left(\nu, \mu_{0}, \sigma_{0}\right)=\left(\kappa_{2}-\kappa_{1}\right)\left(\left(x_{\mu}\right)_{0}\left(m_{\sigma}\right)_{0}-\left(m_{\mu}\right)_{0}\left(x_{\sigma}\right)_{0}\right) \\
& +\kappa_{3}\left(\left(x_{\mu}\right)_{0}\left(y_{\sigma}\right)_{0}-\left(y_{\mu}\right)_{0}\left(x_{\sigma}\right)_{0}\right) \text {, } \\
& \left(\eta_{T N B_{1} B_{2}}\right)_{4}\left(\nu, \mu_{0}, \sigma_{0}\right)=\left(\kappa_{1}-\kappa_{2}\right)\left(\left(x_{\mu}\right)_{0}\left(z_{\sigma}\right)_{0}-\left(z_{\mu}\right)_{0}\left(x_{\sigma}\right)_{0}\right)
\end{aligned}
$$




$$
+\left(\kappa_{3}-\kappa_{2}\right)\left(\left(x_{\mu}\right)_{0}\left(y_{\sigma}\right)_{0}-\left(y_{\mu}\right)_{0}\left(x_{\sigma}\right)_{0}\right) .
$$

Finally, we get

Theorem 12. $\gamma(\nu)$ is a geodesic curve where $T N B_{1} B_{2}$-Smarandache curve $r_{T N B_{1} B_{2}}$ of the curve $\gamma(\nu)$ is isoparametric on the hypersurface $\varphi_{T N B_{1} B_{2}}(\nu, \mu, \sigma)$ in $G_{4}$ if the conditions

$$
\left\{\begin{array}{l}
x\left(\nu, \mu_{0}, \sigma_{0}\right)=y\left(\nu, \mu_{0}, \sigma_{0}\right)=z\left(\nu, \mu_{0}, \sigma_{0}\right)=m\left(\nu, \mu_{0}, \sigma_{0}\right)=0, \\
\left(\eta_{T N B_{1} B_{2}}\right)_{2}\left(\nu, \mu_{0}, \sigma_{0}\right) \neq 0, \quad\left(\eta_{T N B_{1} B_{2}}\right)_{3}\left(\nu, \mu_{0}, \sigma_{0}\right)=\left(\eta_{T N B_{1} B_{2}}\right)_{4}\left(\nu, \mu_{0}, \sigma_{0}\right)=0
\end{array}\right.
$$

are satisfied. Here, $\nu \in\left[L_{1}, L_{2}\right], \mu_{0} \in\left[T_{1}, T_{2}\right], \sigma_{0} \in\left[M_{1}, M_{2}\right]$.

Authors Contribution Statement All authors have contributed to all parts of the article. All authors read and approved the final manuscript.

Declaration of Competing Interests The authors declare that they have no competing interests.

Acknowledgements The authors thank the referee(s) for valuable suggestions which have improved the final version of the paper.

\section{REFERENCES}

[1] Abdel-Baky, R.A., A surface family with a common asymptotic curve in the Euclidean 3space, Asian Journal of Mathematics and Applications, Volume 2016, Article ID ama0363, 12 pages.

[2] Altın, M., Yüzbaşı, Z. K., Surfaces using Smarandache asymptotic curves in Galilean space, International Journal of Mathematical Combinatorics, 3 (2020), 1-15.

[3] Bayram, E., Kasap, E., Hypersurface family with a common isogeodesic, "Vasile Alecsandri" University of Bacau Faculty of Sciences Scientific Studies and Research Series Mathematics and Informatics, 24 (2) (2014), 5-24.

[4] Bayram, E., Güler, F., Kasap, E., Parametric representation of a surface pencil with a common asymptotic curve, Computer-Aided Design, 44 (2012), 637-643, https://doi.org/10.1016/j.cad.2012.02.007.

[5] Bayram, E., Kasap, E., Hypersurface family with a common isoasymptotic Curve, Hindawi publishing Corporation Geometry, Volume 2014, Article ID 623408, 6 pages, https://doi.org/10.1155/2014/623408.

[6] Elzawy, M., Mosa, S., Smarandache curves in the Galilean 4-space $G_{4}$, Journal of the Egyptian Mathematical Society, 25 (2017), 53-56, https://doi.org/10.1016/j.joems.2016.04.008.

[7] Kasap, E., Akyildiz, F. T., Orbay, K., A generalization of surfaces family with common spatial geodesic, Applied Mathematics and Computation, 201 (2008), 781-789, https://doi.org/10.1016/j.amc.2008.01.016.

[8] Kasap, E., Akyildiz, F. T., Surfaces with common geodesic in Minkowski 3-space, Appl.Math. Comput., 177 (2006), 260-270, https://doi.org/10.1016/j.amc.2005.11.005.

[9] Yüzbaşı, Z. K., Bektaş, M., On the construction of a surface family with common geodesic in Galilean space $G_{3}$, De Gruyter Open Phys., 14 (2016), 360-363, DOI 10.1515/phys-2016-0041.

[10] Yüzbaşı, Z. K., On a family of surfaces with common asymptotic curve in the Galilean space $G_{3}$, Journal of Nonlinear Sci. Appl., 9 (2016), 518-523, DOI:10.22436/jnsa.009.02.17. 
[11] Yüzbaşı Z. K., Yoon, D. W., An approach for hypersurface family with common geodesic curve in the 4D Galilean space $G_{4}, J$. Korean Soc. Math. Educ. Ser. B: Pure Appl. Math., 25 (4) (2018), 229-241, DOI:10.7468/jksmeb.2018.25.4.229.

[12] Öztekin, H., Bozok, H. G., Inextensible flows of curves in 4-dimensional Galilean space $G_{4}$, Mathematical Sciences and Applications E-Notes, 1 (2) (2013), 28-34.

[13] Şaffak, G. and Kasap, E., Family of surface with a common null geodesic, Internat. J. Phys. Sci., 4 (8) (2009), 428-433.

[14] Şaffak Atalay, G., Kasap, E., Surfaces family with common Smarandache asymptotic curve, Bol. Soc. Paran. Mat., 34 (1) (2016), 9-20, doi:10.5269/bspm.v34i1.24392.

[15] Wang, G. J., Tang, K., Tai, C. L., Parametric representation of a surface pencil with a common spatial geodesic, Computer-Aided Design, 36 (2004), 447-459, https://doi.org/10.1016/S0010-4485(03)00117-9.

[16] Yllmaz, S., Consruction of the Frenet-Serret frame of a curve in 4D Galilean space and some applications, Int. Jour. of the Phys. Sci. Vol., 5 (8) (2010), 1284-1289.

[17] Yoon, D. W., Lee, J. W., Lee, C. W., Osculating curves in the Galilean 4-space, International Journal of Pure and Applied Mathematics, 100 (4) (2015), 497-506, DOI:10.12732/IJPAM.V100I4.9. 